f/b chemotherapy post-excision.

Conclusion: It is important to consider ovarian cancer in the differential diagnosis for inguinal lymphadenopathy in a female patient. The optimal treatment for these isolated recurrences is unknown. Larger studies are necessary to identify prognostic factors and substantiate apt treatment strategies specifically to this unusual rare clinical condition.

Poster (M14)

Gynecologic Cancer Screening

https://doi.org/10.3802/jgo.2021.32.S1.M14

\section{Predictive value of 'Smartscopy' for detection of preinvasive cervical lesions during COVID-19 pandemic: a diagnostic study}

\section{Thamawoot Phoblap, Pilaiwan Kleebkaow, Bandit Chumworathayi, Sanguanchoke Luanratanakorn, Apiwat Aue-aungkul, Yuwadee Itarat, Amornrat Temtanakitpaisan*}

Department of Obstetrics and Gynecology, Faculty of medicine, Khon Kaen University, Khon Kaen, Thailand (kunglekmed@hotmail.com)

Objective: To evaluate the predictive value of 'Smartscopy' using a snapshot from a smartphone to diagnose preinvasive cervical lesions in patients with abnormal cervical screening. Methods: This study was a diagnostic study. Women with abnormal cervical cancer screening were enrolled at colposcopy clinic in Srinagarind Hospital, Khon Kaen University between September 2020 and March 2021. The cervix was evaluated by using a smartphone and colposcopy by two independent colposcopists. Colposcopy with cervical biopsies and endocervical curettage were performed as standard procedures. The diagnostic performance of the smartphone for cervical intraepithelial neoplasm was assessed. The kappa value was tested to evaluate the correlation of two colposcopists. Results: Two hundred seventy-four patients with abnormal cervical screening were enrolled. There was a significant correlation between histologic diagnoses based on the smartphone and colposcopic findings $(\mathrm{K}=0.88,95 \%$ confidence interval $[\mathrm{CI}]=0.82-0.93)$. The AUC value, sensitivity, specificity, positive predictive value, negative predictive value, and accuracy of the smartscopy to detect CIN1+were 0.54 (95\% CI $=0.51-0.57), 96.6 \%$ (95\% CI $=91.6-99.1), 12.9 \%$ (95\% CI $=8.06-19.2$ ), 46.2\% (95\% CI=39.7-52.4), 83.3\% (95\% $\mathrm{CI}=62.6-95.3), 0.49 \%(95 \% \mathrm{CI}=0.43-0.55)$ respectively. The AUC, sensitivity, specificity, positive predictive value, negative predictive value, and accuracy of the smartphone in the diagnosis of CIN2+ were 0.765 (95\% CI=0.704-0.826), $67.6 \%$ (95\% CI=55.2-78.5), 85.4\% (95\% CI=79.9-90), 60.5\% (95\%
$\mathrm{CI}=48.6-71.6), 88.9 \%$ (95\% CI=83.7-92.9), 0.81 (95\% CI=0.75$0.85)$ respectively.

Conclusion: Smartscopy has a remarkable correlation with colposcopy and high predictive value to detect preinvasive cervical lesions. Therefore, smartscopy demonstrated usefulness as an alternative cervical cancer screening tool in low resource medical setting and had made telemedicine an essential social distancing during covid-19 pandemic.

Poster (M15)

Miscellaneous

https://doi.org/10.3802/jgo.2021.32.S1.M15

\section{Course and pattern of venous thromboembolism in ovarian cancer patients}

\section{Suwanit Therasakvichya, "Wanniga Saengsuri}

Faculty of Medicine, Siriraj Hospital, Mahidol University, Bangkok, Thailand (suwanit.the@mahidol.ac.th)

Objective: Malignancy has been described as a risk factor for venous thromboembolism (VTE) which is shown to significantly affect mortality and morbidity. The objective of this study was to identify the incidence and the course-pattern of VTE in ovarian cancer patients.

Methods: Retrospectively reviewing medical records, from January 2005 to December 2009, of 993 ovarian cancer patients who were diagnosed with the first episode of VTE in the Department of Obstetrics and Gynecology, Siriraj Hospital. Thromboembolic events were confirmed with imaging studies which include duplex-doppler sonography or other radiologic procedures. Data analysis was performed to evaluate the cumulative incidence and course-patterns of thromboembolic events.

Results: A total of 46 ovarian cancer patients were diagnosed of VTE, the mean age was 54.5 years old (range, 33-82). The 5-yearcumulative incidence is $4.63 \%$. VTE occurred $77 \%$ in the lower limbs (common femoral and popliteal veins) and more prevalence on the left side, $15 \%$ in the pelvis, and $17 \%$ in the pulmonary vein. Time-courses (mean + standard deviation) between diagnosis of ovarian cancer and VTE event were 4.2+5.1, 4.8+4.2, and $48.3+56.6$ weeks before ovarian cancer diagnosis, during ovarian cancer treatment, and aftertreatment or proceeding recurrence disease respectively. Two of symptomatic pulmonary embolism cases were fatal after surgery.

Conclusion: The 5 year-cumulative incidence of VTE in ovarian cancer patients is $4.63 \%$. Symptomatic pulmonary embolism had been identified to have a negative impact on survival. Awareness of VTE should be considered during the course treatment of ovarian cancer patient. 